\title{
A New Tier Based Approach for Clustered Content Centric Networks Implementing Cloud Computing
}

\author{
W. A. Gulzar, M. U. J. A Sundhu, and A. R. Butt
}

\begin{abstract}
Since last four decades, internet exploration and innovation has touched new horizons but all that advancement in realm of internet was based on peer to peer interaction or mostly client server topology. There were many loop holes in such configuration as all the communication was machine identified and all the available resources in the network were not fully utilized, therefore a more vigorous content centric model was proposed and is expanding at a very fast pace in which all the data is named and could be searched on the basis of name, instead of machine address. There are many open challenges faced by content centric networks, one is naming of huge amount of data available and efficiently addressing this data dynamically and caching of huge information and its fast addressing. The other issue of importance is congestion control where no host to host based communication is taking place and the nodes are searching and transferring required data in an independent fashion. The economical solution to the problem of global deployment of this new technique. In our approach, we have suggested and implemented a new model that provides remedies for current bottlenecks in $\mathrm{CCN}$ by incorporating the advantages offered by cloud computing with little overheads. We have divided content centric networks in the form of tiers based on the similarity of information that they handle every day in close vicinity, the tiers are further divided in to clusters whose size and number would be dependent upon the number of users. The new approach has been given the names clustered $\mathrm{CCN}$ or $\mathrm{CCCN}$.
\end{abstract}

Index Terms-Clustered content centric networks, CCN.

\section{INTRODUCTION}

Internet based communication was originally designed for host to host or peer to peer with every machine on the internet having unique identifiers and all the communication and routing is address based. Most of the communication on internet is client server based e.g. World Wide Web is providing its server based services to most of the internet users worldwide to date.

This scheme is though simple and conventional but has not capitalized the full capacity and potential of the network and many of available resources are not properly utilized especially when they can be easily available locally. A most common example is that Alice wants to get a certain MP3 file from a remote server or a copy of it may be placed at a local $\mathrm{CDN}$, if that is a popular and most visited Server or CDN then Alice has to wait for a remotely placed, busy location whereas the same information may have been downloaded by an adjacent machine in the same local network that may be easily

Manuscript received February 2, 2014; revised March 27, 2014.

The authors are with Mirpur University of Science and Technology, Mirpur AJK 10250, Pakistan (e-mail: wahab.ali@must.edu.pk, umairsundhu@gmail.com, asifrazabutt@gmail.com). accessible as compared to the previous situation. Most of internet today is based on content distribution e.g. file exchange, audio video streaming etc. that are placed usually at popular servers and is mostly dependent on the location where the information is currently placed. Though the use of CDN's has helped in this regard but they prove to be expensive and also ensuring their availability is crucial. Also security related issues arise regarding content easily misplaced over ordinary unsecure connections. Different authentication and confidentiality measures need to be adopted on node to node interaction level, whereas if data itself is authenticated as in named content technique, need for authentication and formation of VPN's is eliminated and more suitable and reliable security measure based on data itself can be introduced which will be free from hackers and intruders intervention. Another issue related to conventional IP based network is the restriction of mobility as we bind a host to its location rather than by the data it contains. As current internet tends to adopt to mobility at a very rapid rate so the ordinary use of patching devices with their addresses (either IP addresses or MAC addresses) results in rigorous network forwarding based on the location of information where the host is placed rather than the information name itself. Name content centric Networking was introduced few years back to propose the shape of future networks and rectify the legacies of the current TCP/IP based communication model. Instead of IP node the basic entity, named data is the central nexus. $\mathrm{CCN}$ architecture consists of named data layout, caching within the network and transmission dependent on the receiver [1]-[3]. First an interest packet is forwarded that may contain the name of particular data required or a prefix of named data dependent on whatever convention is used in the network from a host that want to retrieve some data. The network is equipped with intrinsic cache systems that are sometimes embedded with intermediate routers. The routers used are specialized routers which search and forward the content based on the content name. The forwarded interest packet would traverse through the network and on finding a match first authenticates and requests the remote node that may be a cache or any other machine containing the data to send that data back to the sender that has initiated the interest packet. Now the receiver would forward the data packet to the sender that is intermediately stored by routers or other allowed storage machines as it travels back. The data packets traverse the same path as that of interest packet. The intermediate storage is for future use of that particular data from a local location that was recently retrieved. The data is stored along with the version and timing in order to update if a fresh copy is available. There are three data structures held by each CCN node namely content store (CS), pending interest 
table (PIT) and forwarding information base (FIB). The request generated called interest for a particular packet is first searched in the local content store and if a match is found, it is forwarded locally, if a match is not found then it is searched in Pending interest table and if a match is found, meaning already a pending request for same data is there, then the interface is the PIT is updated so that when the required data is found, it would be forwarded to all the pending interfaces. If no match is found in PIT then it is forwarded to FIB, for a match. If a match is found, a new entry is made in PIT and the interest is forwarded. The path of data return is the same as that of the interest. Thus the major advantages gain in such kind of architecture are reduced congestion owing to content caching, better delivery speed, basic and simple configuration nomenclature for network devices and providing security at data level instead of network devices based [4]. Comparison of $\mathrm{CCN}$ with conventional TCP/IP stack. ( See Fig. 1).
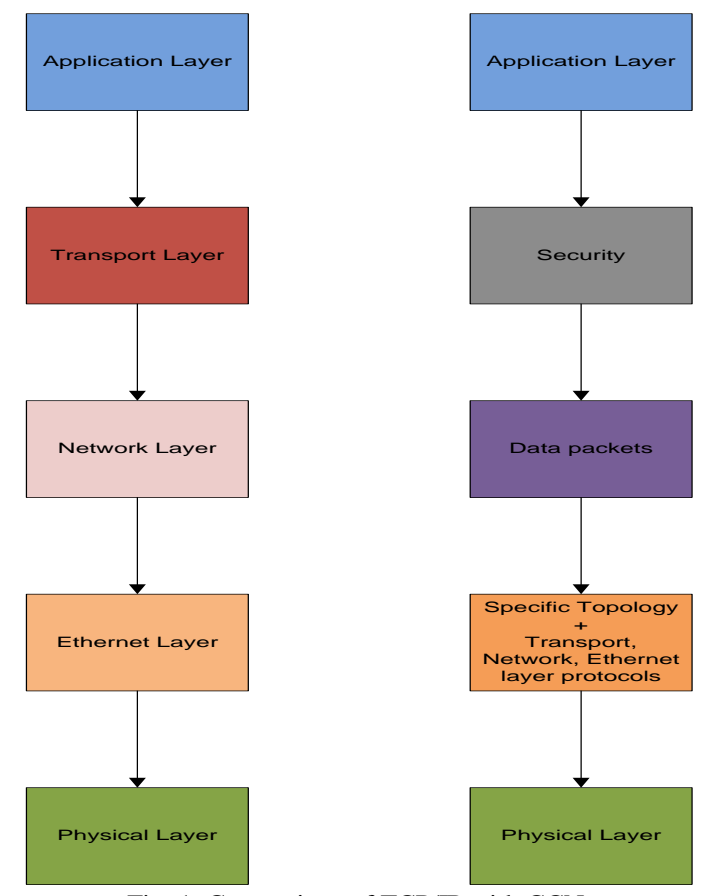

Fig. 1. Comparison of TCP/IP with CCN.

Most of the layers have an agreement with layer 3 requiring universal agreement and showing that $\mathrm{CCN}$ can be tailored over any layer mostly over IP layer. The major difference is at Specific Topology and security that departs from the typical IP networking configuration [5], [6]. Packet format for interest and data packet format in $\mathrm{CCN}$ is depicted in Fig. 2.

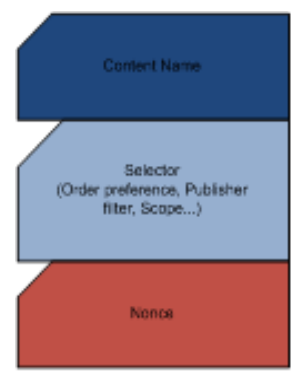

Interest packet

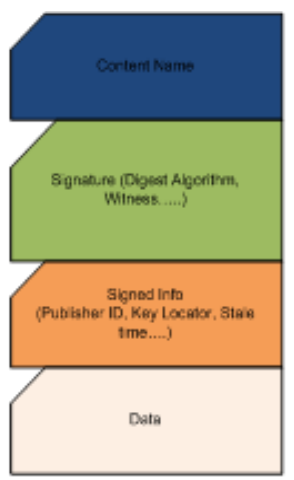

Data packet
Fig. 2. CCN packet format.

\section{Cloud COMPuting}

Cloud Computing concept was introduced about a mere decade ago and since then it has seen a tremendous positive response due to the availability of virtual servers and open source utilization. The offering of application service provision at a remote location has led to the introduction of almost all sorts of solutions online ranging from hardware to software. The remote renting of resources helps especially in the paradigm of information storage anywhere without the need to actually buy hardware and its corresponding maintenance and related software update. The three platforms generally offered under the umbrella of cloud computing are Software as Service, Platform as Service, Infrastructure as service. This concept of remote handling of provisioned resources can prove very helpful in content centric networks [7], and in our model we will use the caching of resources via cloud in order to reduce cost and effort for installation of new hardware especially for base controllers in our project that would be located at the edge of each tier.

\section{PROBLEM IDENTIFICATION}

Though content centric networking offers a very handy option for an alternate approach towards our current internet services but there are many challenges in its effective ubiquitous implementation [8], [9]. There are four basic problems that we have tried to rectify in our new approach. Those problems include global scalability, cache management, and Congestion control and deployment issues. There are about more than of the order of $10^{5}$ unique data objects worldwide that needs to be named and a robust and efficient routing as well as resolution system is required in order to provide global scalability. The major problem in Cache management is distributed storage capacity, effective addressing of data and interconnectivity of different caches. The CCN architecture is receiver dependent and the end to end host communication rules are not obeyed as in conventional architecture, so in case of congestion the end hosts cannot throttle the data flow or apply flow control. Deployment of new $\mathrm{CCN}$ based architecture requires interest from users, business community and other technical support issues relating to scale of deployment based on type of traffic flowing in vicinity, information reuse model statistics, all need to be covered, without which this technique may prove very costly and alien to current users and ISP's. Switching to this new scheme requires economic incentives to investors in order to make this scheme more attractive.

\section{Methodology}

The basic methodology of project is to divide the global access into multiple tiers and clusters and the new technique is given the name Clustered $\mathrm{CCN}$, those tiers are not the conventional tiers as we interpret in terms of internet service providers. These tiers are formed on the basis of level and type of data that is generated in particular vicinity. Those tiers may be formed by users independent of ISP's, by simply joining based on the type of information they require mostly. But we have divided for simplicity our $\mathrm{CCCN}$ into three tiers. 
Increased granularity at base level would ensure better handling of named contents. Numerous clusters would be formed within a particular tier based on the number of users and connections in the near vicinity. At the edge of each tier, a base controller is introduced that would be similar to a CDN. Each tier would have a special search preference within it before passing through base controller for search of data into another tier. A particular tier form a type of Adhoc configuration with the advantage of fast searching and matching as special identifiers are appended along with the name prefix.

Tier-I consist of ordinary customers, that are either mobile or immobile or Adhoc based. In Tier-I There could be multiple clusters but for simplicity we have considered only one cluster per tier. The advantage of cluster is that with in a tier, data is first searched in the current cluster and then adjoining clusters of a particular tier. For a $\mathrm{CCN}$ interest request generated by any of the hosts with in a cluster, it would first be routed within that particular cluster of that tier. The routing scheme for each tier would be different. If nothing found in the first tier of cluster of machines, it would be forwarded to next tier through a base controller that would be monitoring the requests flow and data between adjacent tiers. This approach would be similar to Tier based cluster of Adhoc nodes with the only exception that once an interest is not successful in a particular tier, it would be routed via a central Base Controller of that particular tier that would update its information after every successful data retrieval from the other tier. In our topology we have supposed that ISP has no restriction on the storage of data in any particular device or networking node. In a particular tier, the data naming would be local and would be differentiated from the other tier by means of identifier in each particular tier that would be unique, though the name of data would be the same but only the addressing and routing convention would be simplified by appending a particular identifier with the naming prefix in the order of hierarchical name that can simplify the search for similar data routes and would be included in the scope area of the interest packet. This technique can ensure a fast data routing based on the prefix identifier of that particular tier. When a particular tier is unsuccessful in retrieving data, the timer would live on and would be readjusted at the base controller along with appending new prefix identifier for the next tier and removing the previous identifier. The same cycle is repeated for the second and third tier. The second tier would be a broad based tier whose cluster would cover up to a particular country level. When the interest request arrives in tier-II, it would be processed in the same fashion and if the data is not found in tier-II then it would be forwarded to tier-III by passing through another base controller. The introduction of base controller ensures that even if a device that may have the copy of information is not active then ultimately based controller can provide the data, if it was previously requested via it as it updates its database on each request through it, so in this fashion not only nodes in clusters store passing data after authentication but also a central base controller of each tier also provides back up. The base controller at any particular tier on retrieval of required data from higher tier would remove the upper level identifier and would route it to the conjurer of interest packet, keeping record of the copy to itself also. Tier-III would be the largest including servers of large enterprisers. A basic form of first tier based cluster is shown below in Fig. 3.

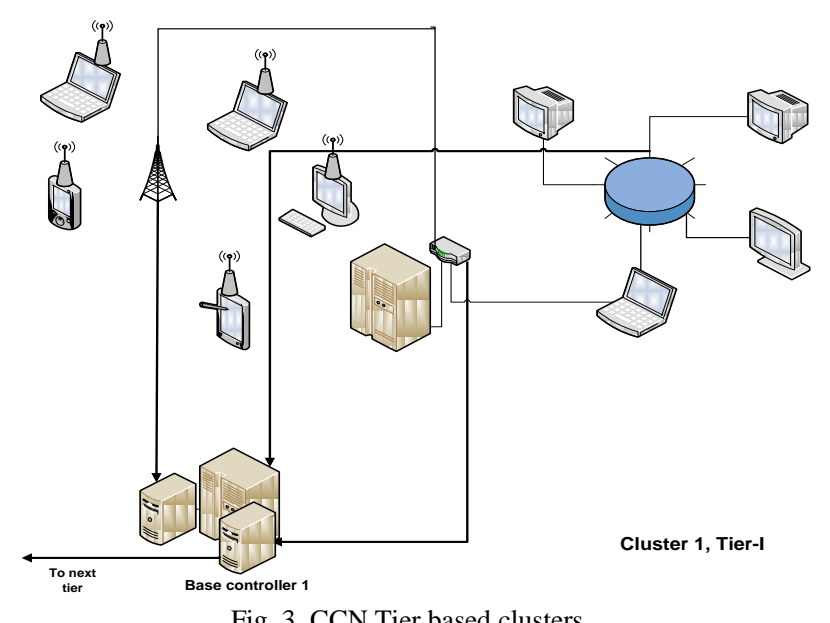

\section{A. Base Controller}

The use of base controller is pivotal to the approach as it would be required if a particular request/interest is not found in a particular tier. The memory size of base controller depends on the tier end where it is located e.g. the lowest tier would have the small memory and database requirements as compared to higher tier base controller. The traffic at any base controller can be modeled as

Based on the popularity of content, the jth request probability is given by

$$
P_{j}=(1 / j) / \sum_{j \varepsilon C 1 / j}
$$

where $C$ is the content requested, $P_{j}$ is the probability of that content.

The objects that are to be requested at hierarchal base controllers have the probability

$$
P_{h}=\sum_{j \varepsilon C x} P_{j}
$$

where $P_{h}$ is the hierarchal probability of content $C_{x}$, that was not available in the particular tier before base controller. If we denote $T_{k}{ }_{k}$ represent the network traffic that is originated for data objects $O_{d}$ request from the tier to its base controller in the vicinity, then

$$
T_{k}^{i}=\left(1-Y_{i k}\right) \cdot R_{k}^{i}\left|O_{d}\right| \cdot \min \{C(N)\}
$$

where $Y$ is the individual element at Base controller, $R_{k}^{i}$ is the number of object $O_{d}$ requests. $N$ represents the individual nodes in a tier which have been searched for content $C$.

The cumulative traffic of an individual Base Controller is modeled as

$$
D=\sum_{i=1}^{M} T_{k}^{i}
$$

where $M$ is the number of nodes in a particular tier. 
Efficient lookup at the base controller would be possible as the requests possibility/probability to the higher level is low as most interests would be entertained at either the cluster level or lower base controller and so would the look up time and complexity and the higher tier edge base controllers would have small number of interest hits so managing the higher memory base controllers would be eased as there would be less traffic through them.

The basic flow graph of interest packet forwarding is shown below in Fig. 4.

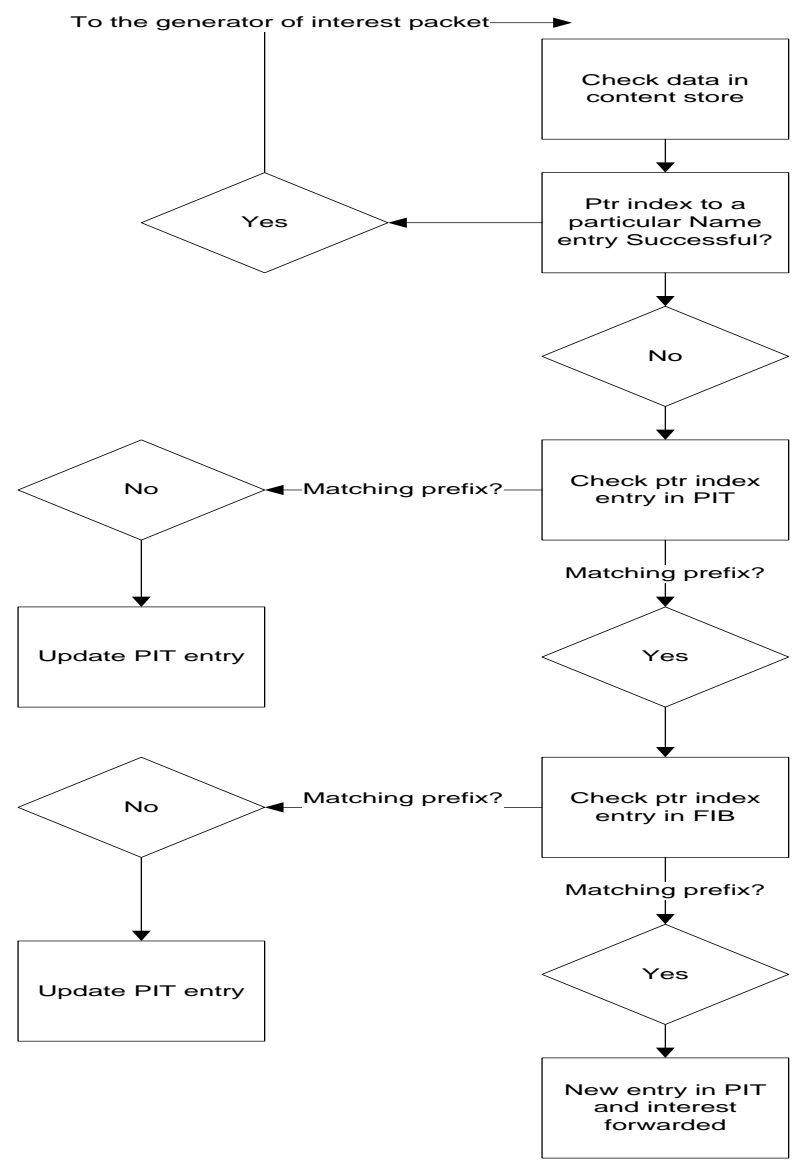

Fig. 4. Flow chart of CCN packet forwarding in a particular tier

As can be seen, the basic mechanism of forwarding is similar to the conventional $\mathrm{CCN}$ architecture, with the only difference that the prefix contains an additional identifier for a particular tier which helps in identification of interest being generated by which tier and its current status, as the tiers hierarchy is predefined. In case of failure of prefix match in CS, PIT, FIB, along with updating the PIT, the interest packet would not time out. The time out mechanism has been extended in this scheme to the point of final base controller which will be lying at the end of tier III which will only discard the interest packet. So, in this project, the size of buffers at intermediate routers is kept large. The global scalability problem can be reduced with fast routing by dividing network in to tiers containing clusters of network nodes. Within a particular tier, routing and naming convention is local so a fast speed overlook could be performed. We are currently working on a fast naming based routing algorithm for intermediate routers that would further suite our topology. The problem of cache management is also resolved owing to fact that we have divided our approach in the form of clusters of nodes in a particular tier and the data stored in them is mostly repeated so no need for huge cache management for individual devices. Also the introduction of one central cache management at base controller can provide a huge cache of local files accessed. Use of specific prefix for a particular tier helps in fast registering and matching indexes even with long sequences in cache search. The issue of deployment is solved by introducing cloud computing and the services of virtual network devices help to encompass the location per resources issues. The platform used in this case is infrastructure as services of cloud computing that would include not only support for heavy cache storage at base controller levels but also the virtual realization of specialized named based routers at intermediate tiers.

\section{B. Cluster Based Identifier in Naming Prefix}

In case of $\mathrm{CCN}$, longest match of prefix is used for any look up. As there is huge number of naming prefixes all over the internet so introducing all of them in any particular router FIB is not possible so a scaled approach is required. One of the solutions to minimize the memory requirement for a FIB data structure is by using Bloom algorithm filter as in [10], yet this approach is not sufficient for restriction in the management of entries of FIB. In our approach one benefit of cluster based routing would be that the number of entries in the FIB would be small and introduction of multi-hierarchal structure reduces the length of route. In our approach a particular cluster would introduce a DNS type route search as shown in the Fig. 5 below:

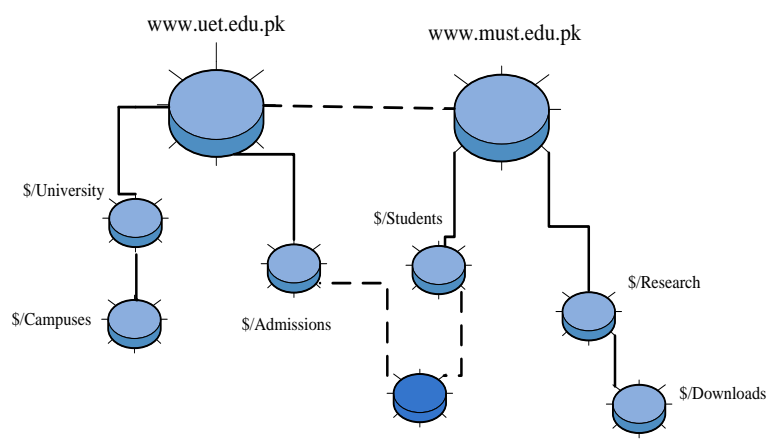

Fig. 5. DNS based route search.

A special identifier would be placed in the hierarchal naming prefix in order to cope up with the scalability problem with in a particular router FIB. Following would the steps in our route formation. In the first phase any particular node search for adjacent neighbors and then introduce the route notification to inform the rest of the nodes about itself and in the later phase FIB entries are constructed cluster-wise, that is done by aggregating the nodes that are searched in the first phase and by using this aggregation the size of entries in a particular FIB would be significantly reduced. In aggregation those entries that have the same route outgoing would be combined into a single entry instead.

\section{Cache Management}

The cache management in $\mathrm{CCN}$ can be divided in to main categories, one is the in network cache and the other is the edge cache. In our topology the edge cache would be the base controller. The in network cache would refer to the local 
cache at the router. The information/content objects are arranged in the form of binary search tree. The content store at any router in a particular cluster would contain a packet storage that is indexed to accelerate to process of matching. In order to keep in view the requirement of popular content demand, the indexing is modified with the inclusion of a popularity counter index that would increase with the number of recent hits and would have the high priority in search. This approach can result in low access time due to small coverage and inclusion of popularity index. The insertion/update, look up and deletion of content is governed by binary search tree algorithm. Within cache the content objects are stored and replaced in accordance with the policies of both Least Recently Used (LRU) and Least Frequency Used (LFU) discussed in [11].

\section{Congestion Control}

In case of content centric networks, simple transport layer protocols that have the tendency to cope up with the problem of congestion cannot be introduced as the data/content object can be retrieved from multiple sources and there is no particular RTT that could be defined and time outs cannot be defined discretely. In [12], [13] the retransmission timer for interest is calculated from the previous data objects as samples, whereas in case of [14] for each data source a particular time out is maintained but the underlying assumption that the receiver knows the location of every data source even before the transfer and there is no change of location during the transfer that is not possible as the $\mathrm{CCN}$ requests could be fulfilled by multiple sources. The introduction of cluster based traffic increases the probability of single time out value as the caches would be close to each other. Other factors that reduce congestion in our topology are effective cache management, reduced traffic in a particular cluster. This topology performs well for congestion cases that are originated mostly from single source. For the case where the traffic is originated from multiple sources, content collection from multiple sources is taken into account and would require a premature or future anticipated interest packet calculation and calculating individual timeouts from each particular source.

\section{E. Router Dynamics}

In conventional routers there are two memory architectures namely multicasting and multi-matching. In multicasting there is forward information base, multicast expansion table and adjacency table. Forwarding information table holds the address of source and information of multicast ring. Adjacency table has the rewrite multicast expansion table index and MAC those are memory types and have a finite capacity. In case of multi-matching, the destination address is compared with the forwarding entry in the table and the longest match is the resultant prefix match. The forwarding table memory is composed of special ternary content addressable memory (TCAM) that is used for high speed search and the resultant search is returned to SRAM by priority encoders. In case of conventional routers, multicasting is done by a match of TCAM entry with the desired address output and the resultant interface is stored in multicast expansion table. In our topology we have used active TCAM and passive SRAM which can ensure a parallel and high speed search.

\section{F. Delay Analysis}

In our technique, introduction of tier based clustering reduces the delay time $t_{d}$ because the hit probability of getting the same information within a particular tier is higher. The second factor is the size of local caches is reduced. Thirdly the introduction of base controller would ensure a swift data retrieval if any such copy has been passed before from the upper tier. Table I shows the parameters for delay calculations.

TABLE I: PARAMETERS FOR DELAY CALCULATION

\begin{tabular}{|l|l|}
\hline$T_{d}$ & Delay time \\
\hline$S$ & Size of cache as per packet places \\
\hline$A$ & Packet sending average time \\
\hline$\mu$ & Content packets arrival rate at router \\
\hline $\mathrm{r}$ & Rate of cache hit \\
\hline $\mathrm{H}$ & Average number of hops \\
\hline $\mathrm{X}$ & Average hop distance \\
\hline $\mathrm{P}$ & Multi-hop probability \\
\hline
\end{tabular}

Queuing delay after one $\mathrm{N}$ hop routers will be

$$
T_{d}(N)=\sum_{c=1}^{s} A /(1-A \mu . \Pi(1-r))
$$

In our technique, the value of $\mathrm{T}_{\mathrm{d}}$ would decrease as the value of the denominator in the above equation would increase, owing to the fact of better cache hits and improvement in the content arrival rates.

The average number of hops reduction in our technique would be

$$
H(n)=\sum_{i=1}^{x}\left(1-P^{i-1} P\right) i / P(n)
$$

The number of hops would be reduced in a particular tier as the tiers are based on similarity of information and so would be the probability of lesser number of hops and greater chance of successful hit after lesser number of hops.

\section{Simulations}

Two test beds were set up with different scenarios to check the performance of our new $\mathrm{CCN}$ topology as compared to the ordinary CCN in OPNET with packet level CCN simulator implemented at the top of TCP/IP in network nodes. The scenario is depicted in Fig. 6. Different file objects of types jpeg, doc, mp3 are retrieved and their performance is measured with the following graphs. The simulation scenario consists of hybrid wired and wireless networks forming clusters. For simplicity, only three clusters are considered with only a single base controller at the edge.

In the first case as shown in Fig. 7. the delay statistics for the convntional and our approach is shown in the differentiator mode and our approach has less delay spread for individual hits and better tuned. In the second case of server performance as shown in Fig. 8. our approach supercedes the conventional approach after some time, as there are some overheads initially but it performs better once 
it has scanned for the neighbourhood and clusters have fully enegized. The average of queing delay is also much less as compared to the conventional approach, the scenario is depicted in Fig. 9. The overall result is high throughput yield in our approach as compared to the conventional approach as is depicted in the last graph Fig. 10.

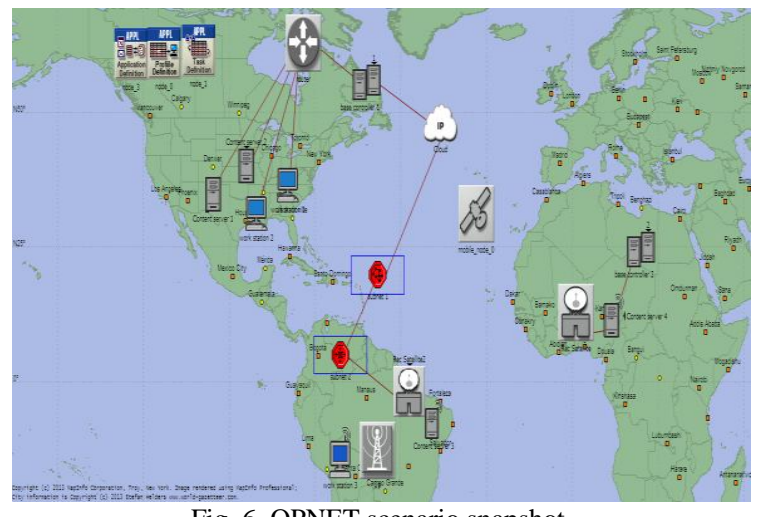

Fig. 6. OPNET scenario snapshot.

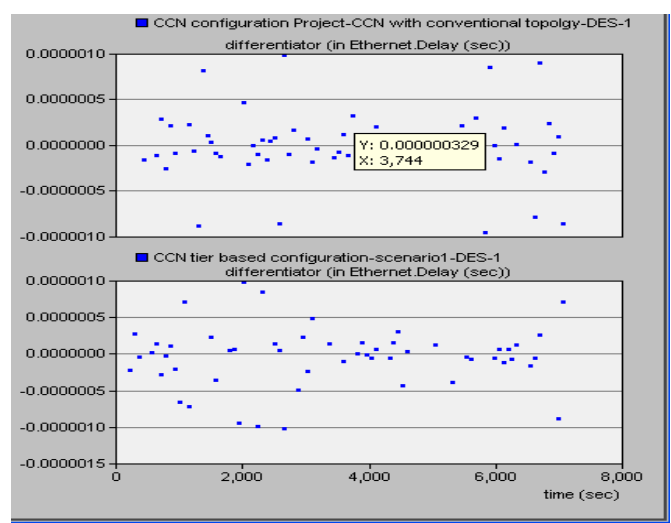

Fig. 7. Delay statistics of two approaches.

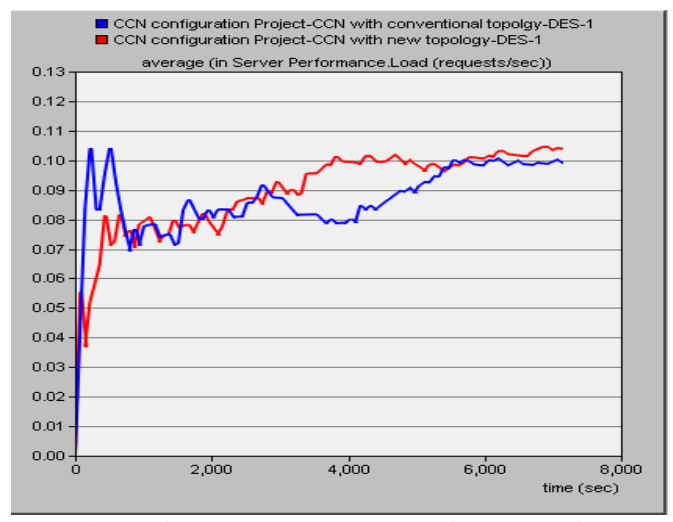

Fig. 8. Comparison of content based server performance of two approches.

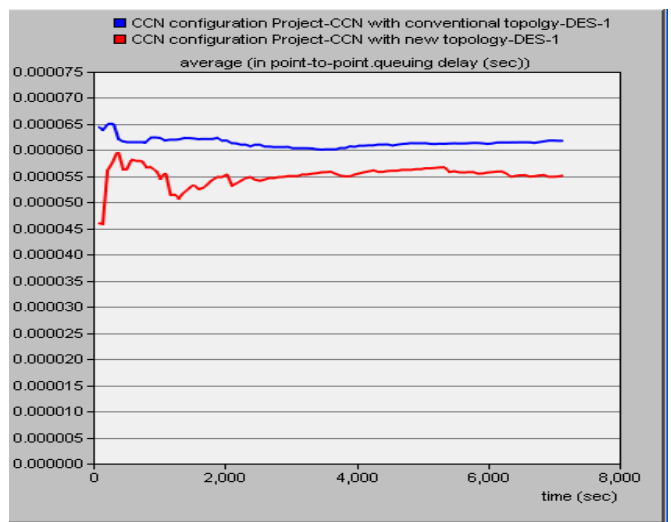

Fig. 9. Point to point queing delay comparison.

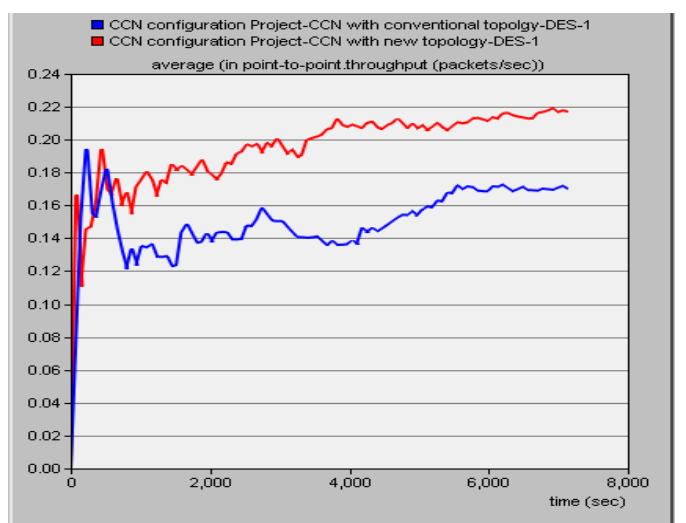

Fig. 10. point to point throughput comparison.

\section{CONCLUSIONS AND FUtURE PlANS}

We have conducted first mathematical based analysis of our technique Clustered CCN or simply CCCN which yielded that our technique performs better in terms of delay and server performance especially when the clusters in the tier are selected on the basis of relevant information. Larger the cluster size, larger the hit probability and better efficiency. Those mathematical calculations are in coherence with the simulation results. A much better performance is yielded as the network is loaded with more interest queries but should not exceed the buffer size capacity as the time to live period of interest packet is extended, The problem of congestion is rare as the size of intermediate router buffers containing CS, PIT and FIB enteries are extended and also due to the introduction of previously mentioned routing technique.

The proposed technique can yield fine results when much finer granuality is ensured at each tier level. The role of base controller is very crucial and gives much better results if distributed cloud computing in terms of infrastructure for storage and maintenance is used instead of integrated approach. Possible ramifications of introducing a short prefix (instead of additional identifer that we have used in this project) that would encompass only a particular tier and based on that short prefix, introducing an adaptive naming routing scheme for each tier is the future course of action.

\section{REFERENCES}

[1] G. Tyson, S. Kaune, S. Miles, Y. El-Khatib, A. Mauthe, and A. Taweel, "A trace-driven analysis of caching in content-centric networks," in Proc. Intl. Conference on Computer Communication Networks, 2012.

[2] A. Detti et al., "CONET: A content centric inter-networking architecture," in Proc. ACM SIGCOMM Workshop on Information-Centric Networking (ICN-2011), Toronto, Canada, Aug. 2011.

[3] V. Jacobson, D. Smetters, J. Thornton, M. Plass, N. Briggs, and R. Braynard, "Networking named content," in Proc. ACM CoNext, 2009.

[4] B. Ahlgren, C. Dannewitz, C. Imbrenda, D. Kutscher, and B. Ohlman. (February 2011). A survey of information-centric networking. [Online]. Available: http://drops.dagstuhl.de/opus/volltexte/2011/2941.

[5] S. Arianfar, P. Nikander, and J. Ott, "On content-centric router design and implications," in Proc. ACM ReArch., Philadelphia, USA, November 30, 2010.

[6] W. Adjie-Winoto, E. Schwartz, H. Balakrishnan, and J. Lilley, "The design and implementation of an intentional naming system," SIGOPS Oper. Syst. Rev., vol. 33, no. 5, pp.186-201, 1999.

[7] B. Ahlgren, P. A. Aranda, P. Chemouil, and S. Oueslati, "Content, connectivity and cloud: ingredients for the network of future," IEEE Communications Magazine, vol. 49, issue 7, Jul. 2011. 
[8] M. Al-Fares, A. Loukissas, and A. Vahdat, "A scalable, commodity data center network architecture," in Proc. ACM SIGCOMM'08, Seattle, WA, USA, Aug. 2008.

[9] T. Koponen et al., "A data-oriented (and Beyond) network architecture," in Proc. SIGCOMM'07, Kyoto, Japan, Aug. 2007.

[10] M. Lee, K. Cho, K. Park, T. T. Kwon, and Y. Choi, "SCAN: Scalable content routing for content-aware networking," IEEE ICC, 2011,

[11] G. Carofiglio et al., "Experimental evaluation of memory management in content-centric networking," in Proc. IEEE International Conference on Communications (ICC), Kyoto, Japan, 2011, pp. 1-6.

[12] G. Carofiglio, M. Gallo, and L. Muscariello, "ICP: Design and evaluation of an interest control protocol for content-centric networking," in Proc. 2012 IEEE Conference on Computer Communications Workshops (INFOCOM WKSHPS), March 2012, pp. $304-309$.

[13] S. Salsano, A. Detti, M. Cancellieri, M. Pomposini, and N. B. Melazzi, "Transport-layer issues in information centric networks," in Proc. the Second Edition of the ICN Workshop on Information Centric Networking, New York, NY, USA, 2012.

[14] S. Arianfar, P. Nikander, L. Eggert, and J. Ott, "Contug: A. receiver driven transport protocol for content-centric networks," in IEEE ICNP, 2010.

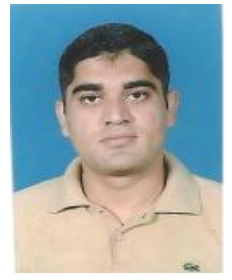

Wahab Ali Gulzar received his bachelor degree in electrical engineering from University of Engineering \& Technology Lahore in 2008 and received his master's degree in electrical engineering from University of Engineering \& Technology, Lahore in 2012 and is currently serving as a lecturer in the Computer System Engineering Department of Mirpur University of Science \& Technology, Pakistan.

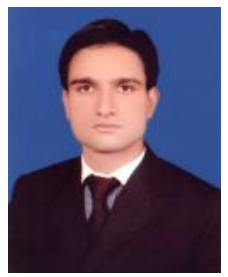

Muhammad Umair Javed Alam Sundhu received his bachelor degree in computer system engineering from COMSATS Abbottabad in 2005 and received his master's degree in electrical engineering from Mirpur University of Science \& Technology in 2009 and is currently serving as an assistant professor in the Computer System Engineering Department of Mirpur University of Science \& Technology, Pakistan.

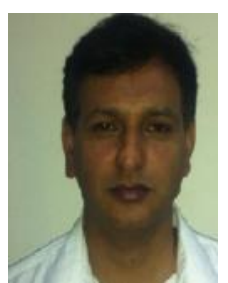

Asif Raza Butt received his bachelor degree in electrical engineering from Azad Jammu \& Kashmir University in 1999 and received his master's degree in electrical engineering from BTH Sweden in 2006 and is currently serving as an assistant professor in the Electrical Engineering Department of Mirpur University of Science \& Technology, Pakistan. 